\title{
The Impact of Lipoprotein-Associated Oxidative Stress on Cell-Specific Microvesicle Release in Patients with Familial Hypercholesterolemia
}

\author{
M. H. Nielsen, ${ }^{1,2}$ H. Irvine, ${ }^{3}$ S. Vedel, ${ }^{4}$ B. Raungaard, ${ }^{5}$ H. Beck-Nielsen, ${ }^{6}$ and A. Handberg ${ }^{2,7}$ \\ ${ }^{1}$ Danish PhD School of Molecular Metabolism, University of Southern Denmark, 5000 Odense, Denmark \\ ${ }^{2}$ Department of Clinical Biochemistry, Aalborg University Hospital, 9000 Aalborg, Denmark \\ ${ }^{3}$ Department of Medicine and Cardiology A, Aarhus University Hospital, 8000 Aarhus, Denmark \\ ${ }^{4}$ Department of Radiology, Aarhus University Hospital, 8000 Aarhus, Denmark \\ ${ }^{5}$ Department of Cardiology, Aalborg University Hospital, 9000 Aalborg, Denmark \\ ${ }^{6}$ Department of Endocrinology M, University of Southern Denmark, 5000 Odense, Denmark \\ ${ }^{7}$ Department of Clinical Medicine, Faculty of Medicine, Aalborg University, 9000 Aalborg, Denmark
}

Correspondence should be addressed to M. H. Nielsen; mohn@rn.dk

Received 5 October 2015; Accepted 31 December 2015

Academic Editor: Domenico D’Arca

Copyright (C) 2016 M. H. Nielsen et al. This is an open access article distributed under the Creative Commons Attribution License, which permits unrestricted use, distribution, and reproduction in any medium, provided the original work is properly cited.

Objective. Microvesicles (MVs) are small cell-derived particles shed upon activation. Familial hypercholesterolemia (FH) particularly when associated with Achilles tendon xanthomas (ATX) predisposes to atherosclerosis, possibly through oxLDL$\mathrm{C}$ interaction with the CD36 receptor. To investigate the hypothesis that MVs derived from cells involved in atherosclerosis are increased in FH and that CD36 expressing MVs (CD36+ MVs) may be markers of oxLDL-C-induced cell activation, cell-specific MVs were measured in FH patients with and without ATX and their association with atherogenic lipid profile was studied. Approach and Results. Thirty FH patients with and without ATX and twenty-three controls were included. Plasma concentrations of MVs and CD36+ MVs derived from platelets (PMVs), erythrocytes (ErytMVs), monocytes (MMVs), and endothelial cells (EMVs), as well as tissue factor-positive cells (TF+ MVs), were measured by flow cytometry. Total MVs, MMVs, EMVs, ErytMVs, and TF+ MVs were significantly increased in FH patients, compared to controls. CD36+ MVs derived from endothelial cells and monocytes were significantly higher in FH patients and oxLDL-C predicted all the investigated cell-specific CD36+ MVs in FH patients with ATX. Conclusions. MVs derived from cells involved in atherosclerosis were increased in FH and may contribute to elevated atherothrombosis risk. The increased cell-specific CD36+ MVs observed in FH may represent markers of oxLDL-C-induced cell activation.

\section{Introduction}

Familial hypercholesterolemia (FH) is an autosomal dominant genetic disorder associated with elevated levels of lowdensity lipoprotein-cholesterol (LDL-C), cholesterol deposits in tendons (xanthomas), and premature heart disease $[1,2]$. The presence of tendon xanthomas (TX) is a marker of increased cardiovascular disease risk among patients with $\mathrm{FH}$ $[3,4]$. Although the underlying mechanisms are not entirely understood, proatherogenic modifications of lipoproteins such as oxidized LDL-cholesterol (oxLDL-C) have been attributed to important roles in both TX development $[5,6]$ and scavenger receptor CD36-mediated atherosclerosis [710]. The activating/cytotoxic effect of oxLDL-C has previously been described [11, 12] and more recent studies demonstrate a relationship between proatherogenic factors, including oxLDL-C, and the shedding of submicron membrane vesicles [13-16]. These cell-specific microvesicles (MVs) are shed from the membrane of activated circulating and vascular cells $[17,18]$ and have in recent years received attention due to their potential involvement in inflammatory and autoimmune diseases, as well as in cardiovascular disorders and the metabolic 
syndrome [19-22]. They express markers from their parental cell and harbor membrane and cytoplasmic proteins as well as bioactive lipids implicated in a variety of cellular mechanisms [23]. Circulating MVs may therefore be looked upon as biomarkers, providing information about their cellular origin as well as the condition of the parental cell, and as active players in disease development and progression [24-26]. CD36 is expressed on various types of cells involved in processes leading to atherosclerosis.

We hypothesize that circulating MVs derived from cells involved in atherosclerosis development are increased with elevated atherosclerosis risk in $\mathrm{FH}$. In particular, we propose that MVs expressing CD36 may be markers of oxLDLC-induced cell activation potentially leading to cholesterol accumulation in the vessel wall. To explore this hypothesis, circulating cell-specific MVs were measured in hypercholesterolemic FH patients with and without Achilles tendon xanthomas (ATX). Furthermore, the potential association between oxLDL-C and the presence of cell-specific CD36positive MVs was studied.

\section{Materials and Methods}

2.1. Study Subjects. The study group comprising thirty patients (18 females and 12 males) genetically diagnosed with heterozygous $\mathrm{FH}$ and twenty-three healthy controls (15 females and 8 males) as indicated by a medical questionnaire has previously been described [6]. FH patients were selected on the presence (11 females and 5 males) or absence (7 females and 7 males) of ATX, according to medical records. None of the participants were taking medication at inclusion, except lipid-lowering therapy, which was withdrawn 8 weeks before study start. The study was conducted in agreement with the Helsinki II declaration and approved by the Central Denmark Region Committees on Health Research (20100147) and by the Danish Data Protection Agency (j. number 2010-41-4879). The presence of ATX was confirmed by ultrasonographic measurements as described in [6].

Blood pressure, height, and body weight were recorded and blood samples were obtained in the fasting state. The following parameters were determined in the routine laboratory: platelet, leukocyte, and monocyte counts, as well as hemoglobin levels were determined on a Sysmex XE$5000^{\mathrm{TM}}$ Automated Hematology System in ethylenediaminetetraacetate (EDTA) blood. A Cobas ${ }^{\circledast} 6000$ analyzer (Roche) was used for analysis of glucose, alanine-aminotransferase (ALT), triglycerides, total cholesterol (Total-C), low-density lipoprotein-cholesterol (LDL-C), high-density lipoproteincholesterol (HDL-C), and apolipoprotein B (ApoB) in lithium-heparin plasma. LDL-C concentrations were estimated from the Friedewald formula [27].

2.2. Determination of oxLDL-C Levels. EDTA plasma for measurement of oxLDL-C was stored at $-80^{\circ} \mathrm{C}$ until being assayed. The Mercodia ELISA assay (Mercodia, Uppsala, Sweden) used for oxLDL-C analysis measures both minimally and fully oxidized LDL-C particles. The intra- and interassay coefficients of variation were $5.5-7.3 \%$ and $4.0-6.2 \%$, respectively, according to the manufacturer.
2.3. Flow Cytometric Measurement of Analysis Microvesicles in Plasma. MV subpopulations were detected and quantitated by a previously reported method [28]. Blood samples for MV preparation were collected into sodium citrate anticoagulant at a $3.2 \%(0.105 \mathrm{M})$ final concentration and processed within 1 hour. Platelet-free plasma (PFP) was prepared by serial centrifugations $(10 \mathrm{~min}$. at $1800 \times \mathrm{g}, 15 \mathrm{~min}$. at $3000 \times \mathrm{g}$, and $5 \mathrm{~min}$. at $3000 \times \mathrm{g}$ ), frozen, and stored at $-80^{\circ} \mathrm{C}$ until analysis. For each analysis, $50 \mu \mathrm{L}$ of freshly thawed PFP was transferred to a TruCount ${ }^{\mathrm{tm}}$ tube (BD Biosciences, New Jersey, USA) containing a lyophilized pellet, which releases a known number of fluorescent beads, used for MV quantification following the manufacturer's instructions. Subsequently, MVs were labeled by adding $10 \mu \mathrm{L}$ fluorescein isothiocyanate(FITC-) conjugated Lactadherin $\left(83 \mu \mathrm{g} \mathrm{mL}^{-1}\right.$, Haematologic Technologies Inc., Vermont, USA) [29, 30], characterized by a phosphatidylserine- (PS-) bonding motif $[29,30]$.

To identify the cellular origin of MVs the following fluorescent mAbs to specific cell surface markers were added immediately after Lactadherin-FITC labeling: $8 \mu \mathrm{L}$ Allophycocyanin- (APC-) conjugated anti-human CD41 $\left(6 \mu \mathrm{g} \mathrm{mL} \mathrm{L}^{-1}\right.$ IgG1, $\kappa$ (clone HIP8, BioLegend, San Diego, CA, USA)) was used to detect platelet-derived MVs (PMVs), $5 \mu \mathrm{L}$ antihuman CD235a-APC $\left(3 \mu \mathrm{g} \mathrm{mL}^{-1}\right.$ IgG2b, $\kappa$ (clone HIR2, eBioscience, San Diego, CA, USA)) was used to detect erythrocyte-derived MVs (ErytMVs), $8 \mu \mathrm{L}$ APC-conjugated anti-human CD31 (50 $\mu \mathrm{g} \mathrm{mL}^{-1} \mathrm{IgG1}, \kappa$ (clone WM59, BioLegend)) and $3 \mu \mathrm{L}$ PerCP-conjugated anti-human CD42b (400 $\mu \mathrm{g} \mathrm{mL}^{-1}$ IgG1, $\kappa$ (clone HIP1, BioLegend)) were used to detect endothelial cell-derived MVs (EMVs) which were defined as particles positively labeled by CD31 mAbs (platelet and endothelium surface antigen) and negative for CD42b (platelet surface antigen) [31]. $5 \mu \mathrm{L}$ Phycoerythrin- (PE-) conjugated anti-human CD36 $\left(6.25 \mu \mathrm{g} \mathrm{mL}^{-1} \mathrm{IgG} 2 \mathrm{a}, \kappa\right.$ (clone 5271)) was used to detect CD36-positive MVs (CD36+ MVs) and $20 \mu \mathrm{L}$ Phycoerythrin- (PE-) conjugated anti-human CD142 (12.5 $\mu \mathrm{g} \mathrm{mL}^{-1}$ IgG1, $\kappa$ (clone HTF-1, BD Pharmingen, New Jersey, USA)) was used to detect tissue factor-positive MVs (TF+ MVs).

After $30 \mathrm{~min}$. of incubation $\left(4^{\circ} \mathrm{C}\right.$, in the dark), $250 \mu \mathrm{L}$ $0.22 \mu \mathrm{m}$ filtered PBS was added to each labeled sample. Plasma samples were analyzed immediately after labeling using a BD FACSAria ${ }^{\mathrm{Tm}}$ III High Speed Cell Sorter, which incorporates three air-cooled lasers at 488, 633, and $407 \mathrm{~nm}$ wavelengths, and equipped with BD FACSDiva ${ }^{\text {rw }}$ software (v. 6.1.3). Logarithmic amplification was used for all channels and isotype controls on plasma samples were used as negative controls. For the detection of MVs an initial microvesiclesize gate was established by pilot standardization experiments using a blend of size-calibrated fluorescent beads, with sizes ranging from $0.2-\mu \mathrm{m}$ (Invitrogen, Hellerup, Denmark) to 3.0- $\mu \mathrm{m}$ (Megamix beads $(0.5,0.9$, and 3.0- $\mu \mathrm{m})$, Biocytex, Marseille, France). The upper limit of the MV gate was set just above the size distribution of the $0.9-\mu \mathrm{m}$ beads in an forward (FSC-A) and side scatter (SSC-A) setting (log scale) using the "autogate" function inside the FlowJo ${ }^{\text {in }}$ (v. 8.8.7, Tree Star, Inc., Oregon, USA) software. The lower limit was the noise threshold of the instrument. This threshold was set at 
TABLE 1: Characteristics of study participants.

\begin{tabular}{|c|c|c|c|c|c|c|}
\hline Characteristics & $\begin{array}{c}\text { Controls } \\
(n=23)\end{array}$ & $\begin{array}{c}\mathrm{FH} \\
(n=30)\end{array}$ & $p$ value $^{\mathrm{a}}$ & $\begin{array}{c}\text { FH ATX- } \\
(n=14)\end{array}$ & $\begin{array}{c}\text { FH ATX+ } \\
(n=16)\end{array}$ & $p$ value $^{\mathrm{b}}$ \\
\hline Men/women & $8 / 15$ & $12 / 18$ & NS & $7 / 7$ & $5 / 11$ & NS \\
\hline Age (years) & $47.0 \pm 10.1$ & $45.5 \pm 9.1$ & NS & $43.1 \pm 10.2$ & $47.5 \pm 7.8$ & NS \\
\hline BMI $\left(\mathrm{kg} / \mathrm{m}^{2}\right)$ & $23.7 \pm 3.3$ & $25.5 \pm 5.2$ & NS & $25.1 \pm 4.2$ & $25.9 \pm 5.9$ & NS \\
\hline Systolic b.p. (mm Hg) & $120.2 \pm 10.8$ & $124.9 \pm 14.3$ & NS & $122.4 \pm 13.7$ & $127.1 \pm 14.8$ & NS \\
\hline Diastolic b.p. (mm Hg) & $74.6 \pm 6.7$ & $77.4 \pm 6.5$ & NS & $76.0 \pm 5.5$ & $78.7 \pm 7.1$ & NS \\
\hline Platelet count $\left(10^{9} / \mathrm{L}\right)$ & $239.8 \pm 50.7$ & $248.6 \pm 60.1$ & NS & $247 \pm 55.7$ & $250 \pm 65.4$ & NS \\
\hline Leukocyte count $\left(10^{9} / \mathrm{L}\right)$ & $5.1 \pm 1.2$ & $5.4 \pm 1.9$ & NS & $5.0 \pm 1.6$ & $5.7 \pm 2.2$ & NS \\
\hline Monocyte count $\left(10^{9} / \mathrm{L}\right)$ & $0.4 \pm 0.1$ & $0.5 \pm 0.1$ & NS & $0.4 \pm 0.1$ & $0.5 \pm 0.2$ & NS \\
\hline Hemoglobin $(\mathrm{mmol} / \mathrm{L})$ & $8.7 \pm 0.6$ & $9.0 \pm 0.8$ & NS & $9.1 \pm 1.0$ & $8.9 \pm 0.7$ & NS \\
\hline Total-C (mmol/L) & $5.1 \pm 0.7$ & $9.1 \pm 1.8$ & $<0.0001$ & $8.2 \pm 1.1$ & $9.9 \pm 2.0$ & 0.0098 \\
\hline LDL-C (mmol/L) & $2.9 \pm 0.5$ & $7.0 \pm 1.8$ & $<0.0001$ & $6.0 \pm 1.1$ & $7.9 \pm 1.8$ & 0.0025 \\
\hline $\mathrm{HDL}-\mathrm{C}(\mathrm{mmol} / \mathrm{L})$ & $1.7 \pm 0.4$ & $1.5 \pm 0.4$ & 0.014 & $1.5 \pm 0.5$ & $1.4 \pm 0.4$ & NS \\
\hline oxLDL-C (U/L) & $50.0 \pm 11.8$ & $100.3 \pm 24.2$ & $<0.0001$ & $83.7 \pm 13.6$ & $114.8 \pm 22.1$ & 0.0001 \\
\hline ApoB (g/L) & $0.8 \pm 0.1$ & $1.7 \pm 0.4$ & $<0.0001$ & $1.4 \pm 0.3$ & $1.8 \pm 0.3$ & 0.0015 \\
\hline Glucose $(\mathrm{mmol} / \mathrm{L})$ & $5.4 \pm 0.5$ & $5.5 \pm 0.4$ & NS & $5.5 \pm 0.4$ & $5.5 \pm 0.4$ & NS \\
\hline $\operatorname{ALT}(\mathrm{U} / \mathrm{L})$ & $19.0(14.0-21.0)$ & $19.0(17.0-34.0)$ & NS & $20.5(18-32)$ & $18.5(15.0-36.5)$ & NS \\
\hline TG (mmol/L) & $1.0(0.8-1.2)$ & $1.1(0.8-1.8)$ & NS & $1.3(0.7-1.8)$ & $1.1(1.0-1.6)$ & NS \\
\hline
\end{tabular}

Normal and nonnormal distributions are shown as the mean \pm standard deviation and as the median (interquartile range), respectively. ${ }^{a}$ Controls versus FH subjects; ${ }^{b} \mathrm{FH}$ subjects without ATX versus FH subjects with ATX. BMI, body mass index; ALT, alanine-aminotransferase, TG, triglycerides; ApoB, apolipoprotein B; Total-C, total cholesterol; LDL-C, LDL-cholesterol; HDL-C, HDL-cholesterol; oxLDL-C, oxidized LDL-C; b.p., blood pressure; NS, nonsignificant.

the SSC-A parameter to prevent exclusion of the smallest events. We defined MVs as particles that were less than $1.0 \mu \mathrm{m}$ in diameter, had positive staining for Lactadherin-FITC, and expressed cell-specific markers. Increased MMV number has previously been reported on the same FH study population [6].

2.4. Statistics. Statistical analyses were carried out using the STATA 11.2 statistical program (StataCorp LP, Texas, USA). MV data were expressed as median (interquartile range) and analyzed using nonparametric Mann-Whitney $U$ tests. Continuous variables with a normal distribution were expressed as the mean \pm standard deviation and analyzed with Student's $t$-test. Statistical correlation was analyzed using Spearman's rank correlation for nonnormal distributed data. Multiple regression (robust) analysis was used to adjust for the confounding effect of gender. The standardized regression coefficients beta $(\beta)$ is included as a measure of how strongly each predictor variable influences the criterion (dependent) variable. Variables with nonnormal distribution were logtransformed to achieve normal distribution before regression analysis. Shapiro-Wilk's $W$ test was used to test the assumption of normality. $p$ values are two-sided and considered significant when $<0.05$.

\section{Results}

3.1. Characteristics of the Study Subjects. Table 1 shows the clinical characteristics of the study participants. Total cholesterol, LDL-C, and oxLDL-C were significantly increased in
FH patients compared to healthy controls, and HDL-C levels were significantly lower. FH patients with ATX had significantly higher concentrations of total cholesterol, LDL-C, and oxLDL-C than FH patients without ATX, whereas HDL-C levels were similar. All other laboratory measurements were within age- and gender-specific reference intervals, and no significant differences were observed between groups.

3.2. Analysis of MV Subpopulations in Patients with FH. FH patients had higher concentrations of total MVs $(p=$ $0.022)$, endothelial cell-derived MVs (EMVs) $(p=0.002)$, erythrocyte-derived MVs (ErytMVs) ( $p=0.043$ ), monocytederived MVs (MMVs) $(p=0.004)$, and tissue factor-positive MVs (TF+ MVs) $(p=0.006)$, when compared to healthy controls (Table 2). No significant difference in plateletderived MVs (PMVs) was observed. As shown in Figure 1, CD36+ MVs were derived from all the investigated cells and most abundantly among MVs derived from platelets, endothelial cells, and monocytes (Table 2). FH patients had higher concentrations of CD36+ EMVs $(p=0.008)$ and CD36+ MMVs $(p=0.001)$, whereas no significant difference in CD36+ PMVs, CD36+ ErytMVs, or total CD36+ MVs was observed. The presence of ATX had no influence on MV levels (data not shown).

3.3. Association between oxLDL-C and CD36-Positive MVs in FH Patients with ATX. OxLDL-C was not correlated with any of the investigated CD36+ MV subpopulations in healthy individuals or in FH patients without ATX. In FH patients with ATX, oxLDL-C showed strong correlations with total 
TABLE 2: Plasma levels of circulating MVs.

\begin{tabular}{lccc}
\hline & Controls $(n=23)$ & FH $(n=30)$ & $p$ value \\
\hline Total MVs & $15603(10549-45211)$ & $35616(23409-58075)$ & 0.022 \\
Total CD36+ MVs & $2161(1274-3213)$ & $2754(1704-3772)$ & NS \\
EMVs & $157(119-238)$ & $282(204-522)$ & 0.002 \\
CD36+ EMVs & $143(85-212)$ & $212(170-408)$ & 0.008 \\
PMVs & $2020(1387-2876)$ & $1664(1382-2773)$ & NS \\
CD36+ PMVs & $1758(1229-2475)$ & $1482(1219-2482)$ & NS \\
MMVs & $5(3-11)$ & $10(7-14)$ & 0.004 \\
CD36+ MMVs & $3(2-4)$ & $5(4-8)$ & 0.001 \\
ErytMVs & $207(147-282)$ & $296(193-453)$ & 0.043 \\
CD36+ ErytMVs & $8(3-13)$ & $11(6-15)$ & NS \\
TF+ MPs & $225(204-396)$ & $408(327-451)$ & 0.006 \\
\hline
\end{tabular}

Values (MVs $\mu \mathrm{L}^{-1}$ plasma) are shown as the median (interquartile range). MVs, microvesicles; MMVs, monocyte-derived MVs; EMVs, endothelial cell-derived MVs; PMVs, platelet-derived MVs; ErytMVs, erythrocyte-derived MVs; CD36, scavenger receptor CD36; TF, tissue factor.

CD36+ MVs $($ Rho $=0.70, p=0.003)$, CD36+ EMVs (Rho $=0.77, p<0.001)$, CD36+ PMVs (Rho $=0.74, p=0.001)$, and CD36+ ErytMVs (Rho $=0.74, p=0.001)$, but not with CD36+ MMVs. No correlation between oxLDL-C and CD36negative subpopulations was observed in any of the three study groups.

Gender-adjusted regression analysis demonstrated strong associations between oxLDL-C and all the investigated CD36-positive MVs, including total CD36+ MVs $\left(R^{2}=0.76\right.$, $p<0.001), \mathrm{CD} 36+\mathrm{EMVs}\left(R^{2}=0.68, p<0.001\right), \mathrm{CD} 36+$ $\operatorname{MMVs}\left(R^{2}=0.41, p=0.027\right)$, CD36+ PMVs $\left(R^{2}=0.64\right.$, $p<0.001)$, and CD36+ ErytMVs $\left(R^{2}=0.51, p=0.003\right)$ in FH patients with ATX (Table 3 ). The association with oxLDLC remained significant for both CD36+ PMVs $\left(R^{2}=0.70\right.$, $p=0.004)$ and CD36+ MMVs $\left(R^{2}=0.41, p=0.034\right)$ after adjusting for platelet and monocyte count, respectively. TF+ MVs and oxLDL-C were significantly associated $\left(R^{2}=0.26\right.$, $p=0.031)$ in FH patients with ATX.

\section{Discussion}

The interest in cell-derived MVs and their potential role in cardiovascular disease (CVD) is steadily increasing, as they may not only reflect the presence of CVD but also play a causative role in its development $[32,33]$. This study shows that $\mathrm{FH}$ patients have significantly higher concentrations of overall circulating MVs and in particular higher concentrations of those derived from endothelial cells, monocytes, and erythrocytes. Moreover, CD36+ MVs derived from endothelial cells and monocytes were significantly higher in $\mathrm{FH}$ patients compared to healthy controls. This study also shows that among FH patients with ATX oxLDL-C was a significant predictor of all the investigated subpopulations of CD36+ MV, whereas MVs negative for CD36 were unrelated to oxLDL-C levels. To the best of our knowledge this is the first study investigating the impact of $\mathrm{FH}$ on level and distribution of circulating MVs, in particular CD36+ MVs, and directly addressing the relationship between plasma levels of proatherogenic oxLDL-C and circulating CD36+ MV subpopulations.
TABLE 3: Multiple regression analysis with oxLDL-C and gender as independent variables.

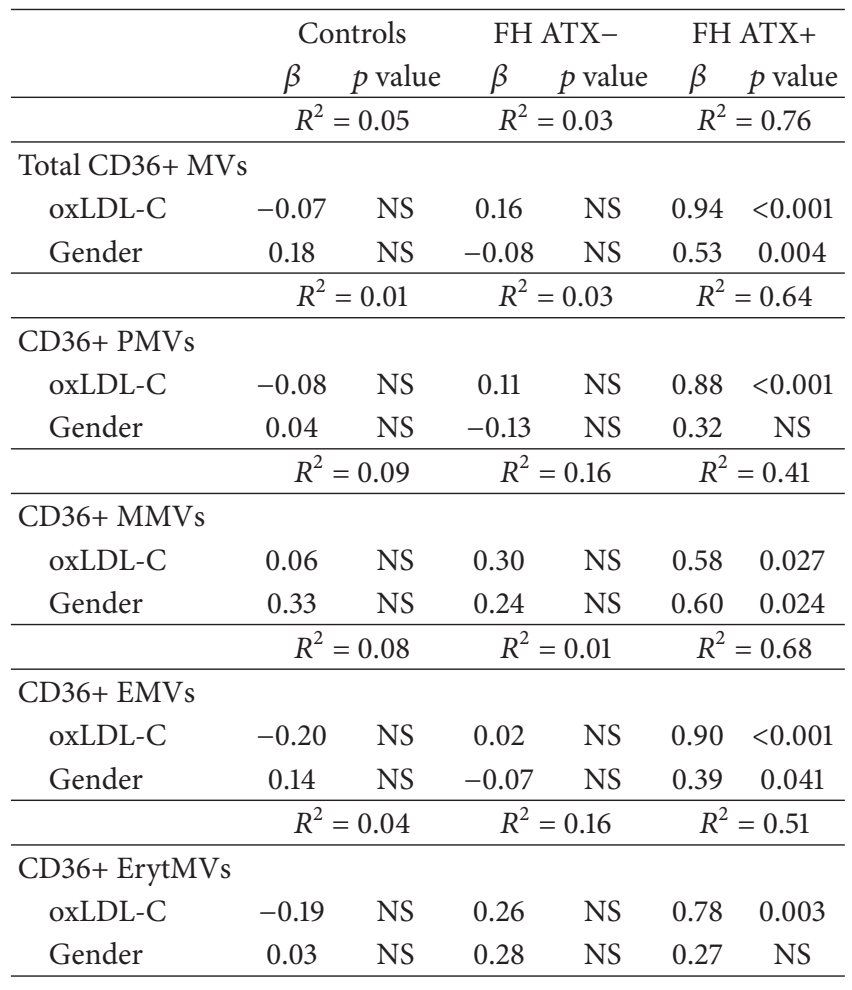

The standardized regression coefficients beta $(\beta)$ is included as a measure of how strongly each predictor variable influences the criterion (dependent) variable. $p$ values are two-sided and considered significant when $<0.05$. See Table 2 for abbreviations.

Elevated LDL-C is a well-known risk factor for atherosclerosis and CVD [34]. Prolonged lipid exposure to cells within the vascular compartments may trigger the release of MVs in concordance with our observation of increased MV concentrations in $\mathrm{FH}$ patients.

This is supported by a recent study demonstrating reduced MV concentrations in $\mathrm{FH}$ patients after an effective lipid-lowering treatment with statins [35]. When comparing 

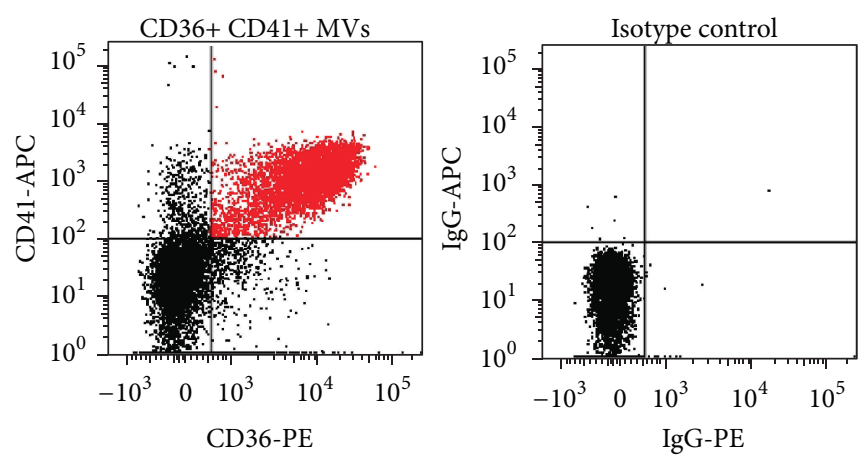

(a)
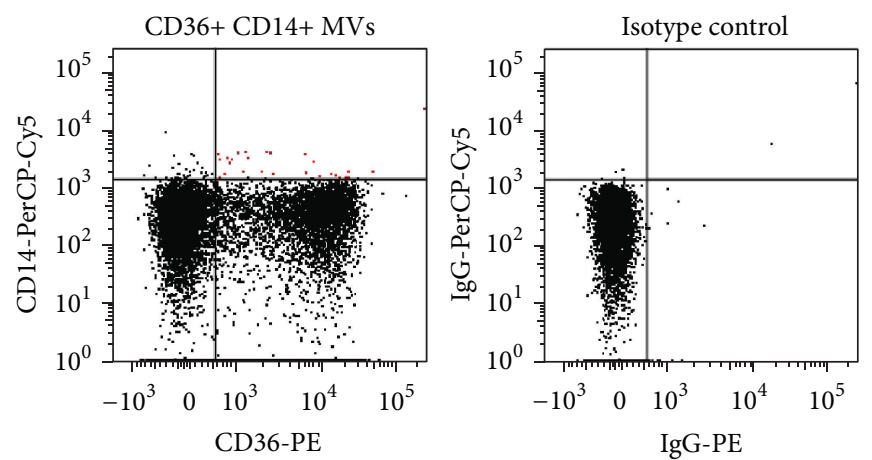

(c)
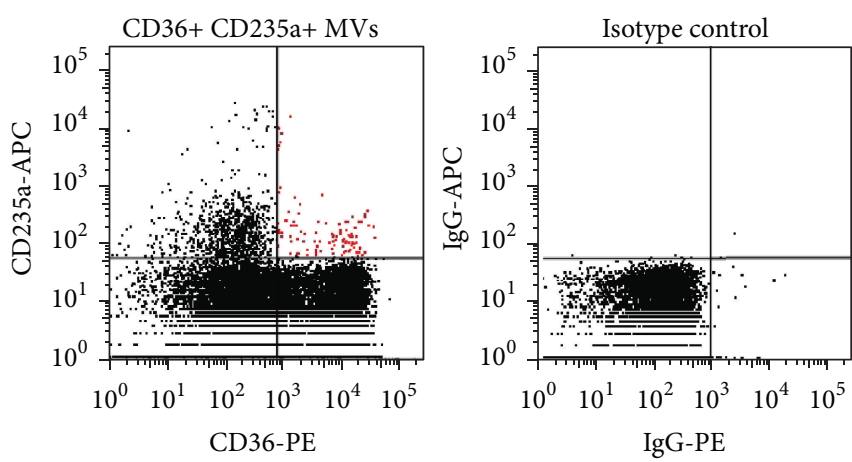

(b)
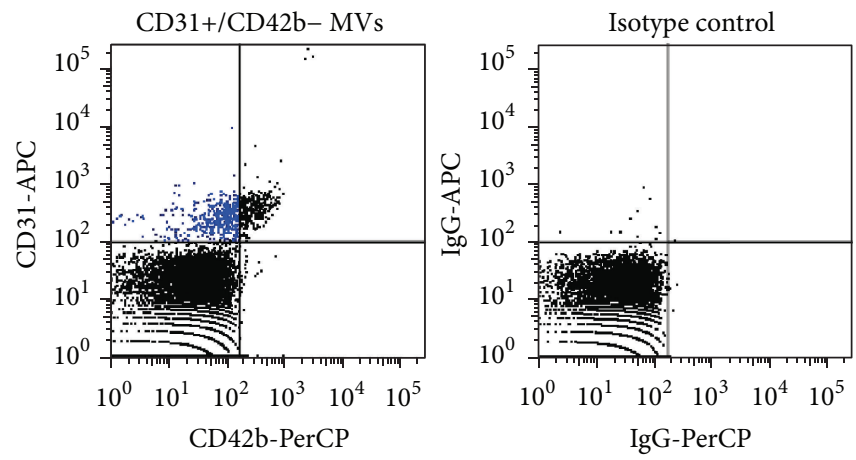

(d)

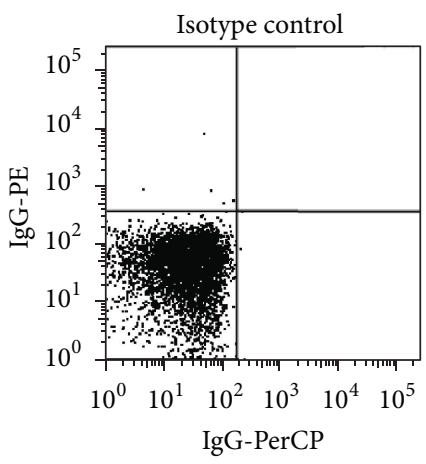

(e)

FIGURE 1: Flow cytometric analysis of CD36-positive MVs derived from various cell types. MVs were identified by size $(<1 \mu \mathrm{m})$ and by surface exposure of phosphatidylserine. Colabeling with cell-specific markers identified CD36 expressing MVs (colored red) from various cell types, including (a) CD36+ CD41+ (platelet-derived) MVs, (b) CD36+ CD235a+ (erythrocyte-derived) MVs, (c) CD36+ CD14+ (monocytederived) MVs, and (d-e) CD36+ CD31+/CD42b- (endothelial cell-derived) MVs identified by a two-color method. CD31 is expressed on both platelets and endothelial cells, whereas $\mathrm{CD} 42 \mathrm{~b}$ is restricted to platelets, allowing discrimination between PMVs and EMVs (colored blue). Right columns correspond to the respective isotype controls.

$\mathrm{FH}$ patients to non-FH hypercholesterolemic patients $\mathrm{MV}$ concentration was higher in the former indicating that both degree and length of exposure affect MV release [36]. Together, these results indicate that overall MVs have the potential of being a marker of hyperlipidemic atherosclerosis risk.

Cell-specific MVs may have some value as markers for the ongoing processes within the vascular compartment, as reported in [23, 37-39]. The finding herein of increased EMV concentrations in FH patients may therefore indicate endothelial cell activation and dysfunction. The increased MMV concentrations most likely reflect activation of inflammatory cells, whereas increased ErytMV concentrations may be associated with prothrombotic risk. Although our study cannot prove causality because of its cross-sectional nature the results presented herein may provide information about the steady-state condition in the vascular compartment during prolonged exposure to elevated LDL-C levels.

Monocyte-derived MVs are like endothelial-derived and erythrocyte-derived MVs significant sources of procoagulant 
activity due to their expression of tissue factor [40, 41], as well as of phosphatidylserine, a cofactor in the coagulation cascade [42]. Elevated levels of monocyte-derived TF+ MVs observed in hyperlipidemia patients are suggested to contribute to arterial thrombosis after rupture of atherosclerotic plaques [42]. Moreover, Wang and colleagues recently demonstrated that monocyte-derived MVs could activate endothelial cells in an IL-1 beta-dependent manner [43]. The observed higher concentrations of both MMVs and overall $\mathrm{TF}+\mathrm{MVs}$ in $\mathrm{FH}$ patients may promote or even accelerate atherothrombosis as suggested in [44].

The association between TF+ MMVs and oxLDL-C has previously been demonstrated in acute coronary syndrome [13]. Moreover, a recent study by Owens et al. reported oxLDL-C-induced TF expression in THP-1 monocytic cells and human monocytes and showed that $\mathrm{FH}$ patients had elevated levels of both oxLDL-C and TF+ MV-associated procoagulant activity [45].

The relationship between serum cholesterol and circulating erythrocytes recently reported in 4,469 adult participants of the National Health and Nutrition Examination Survey (NHANES) [46] suggested that erythrocyte viability is related to cholesterol levels. Erythrocyte membranes contain a ratio of cholesterol and phospholipid, which affects their deformability, and without a nucleus and organelles to synthesize lipids, a large proportion of membrane cholesterol content originates from plasma lipids [47]. Changes in shape and deformability may lead to the production of ErytMVs and increased concentrations in $\mathrm{FH}$ patients, as presented herein. A possible consequence may be activation of the vascular endothelium, resulting in vascular inflammation and atherosclerosis as previously suggested [48].

Proatherogenic modifications of lipoproteins such as the oxidation of LDL-C are attributed to important roles in the atherogenic process. The activating effect of oxLDL-C causing membrane vesiculation has been described in endothelial cells [49], platelets [14], and monocytes [13, 50], and in a previous published work from our group we reported that high numbers of MMVs are associated with proinflammatory monocytes and oxLDL-C [6]. The binding of oxLDL-C to CD36 is considered to trigger intracellular signaling events, but the precise contribution of CD36 is still a matter of debate $[51,52]$. Knowing that CD36 expression is a common feature of these cell types, we propose that oxLDL-C binding to CD36 may lead to cell activation and MV release. The increased $\mathrm{MV}$ concentrations found in $\mathrm{FH}$ patients and the strong association between CD36+ MVs and oxLDL-C in FH with ATX suggest a possible involvement in processes leading to endothelial dysfunction and cardiovascular risk.

This study has some limitations as we used $900 \mathrm{~nm}$ polystyrene microspheres from Megamix to define the upper limit of a forward scatter MV size gate. Although this gating strategy has been published by the ISTH Scientific Standardization Committee $[53,54]$ the difference in refractive index and thus forward angle light scatter between polystyrene beads and cellular MVs must be taken into account, as stated in a very recent study by van der Pol et al. [55].

In conclusion, increased circulating MVs, in particular MMVs, EMVs, ErytMVs, and TF+ MVs, may contribute to atherosclerosis development and elevated atherothrombosis risk in patients with $\mathrm{FH}$. The increased endothelial- and monocyte-derived CD36+ MVs may reflect the state of their parental cells and indicate oxLDL-C-induced activation through a CD36-dependent mechanism. The association between oxLDL-C and CD36+ MVs in FH patients with ATX suggests the involvement of lipoprotein-associated oxidative stress in MV release. Thus, although the prognostic potential of circulating MVs is still in its infancy, our findings indicate that detection and quantification of MV subpopulations, especially CD36+ MVs, may be a potentially valuable tool for risk assessment and may add to a better understanding of their potential contribution to vascular complications in hypercholesterolemia.

\section{Abbreviations

$\begin{array}{ll}\text { ATX: } & \text { Achilles tendon xanthomas } \\ \text { CD36: } & \text { Cluster of differentiation } 36 \\ \text { EMVs: } & \text { Endothelial cell-derived MVs } \\ \text { ErytMVs: } & \text { Erythrocyte-derived MVs } \\ \text { FH: } & \text { Familial hypercholesterolemia } \\ \text { FSC-A: } & \text { Forward scatter area } \\ \text { MVs: } & \text { Microvesicles } \\ \text { MMVs: } & \text { Monocyte-derived MVs } \\ \text { oxLDL-C: } & \text { Oxidized LDL-cholesterol } \\ \text { PFP: } & \text { Platelet-free plasma } \\ \text { PMVs: } & \text { Platelet-derived MVs } \\ \text { PS: } & \text { Phosphatidylserine } \\ \text { SSC-A: } & \text { Side scatter area } \\ \text { TF: } & \text { Tissue factor. }\end{array}$

\section{Conflict of Interests}

The authors declare that there is no conflict of interests regarding the publication of this paper.

\section{Acknowledgments}

All studies were performed at the Department of Clinical Biochemistry, Aarhus University Hospital, and at the FACS Core Facility, the Faculty of Health, Aarhus University, Denmark. The authors thank Anne Lone Larsen for her superb ELISA skills and Charlotte Christie Petersen for her excellent technical assistance with regard to MV determinations. The Danish Heart Association, the Novo Nordisk Foundation, and the Novo Nordisk Foundation via Danish PhD School of Molecular Metabolism supported this study.

\section{References}

[1] D. J. Rader, J. Cohen, and H. H. Hobbs, "Monogenic hypercholesterolemia: new insights in pathogenesis and treatment," The Journal of Clinical Investigation, vol. 111, no. 12, pp. 17951803, 2003.

[2] S. G. Tsouli, D. N. Kiortsis, M. I. Argyropoulou, D. P. Mikhailidis, and M. S. Elisaf, "Pathogenesis, detection and treatment of Achilles tendon xanthomas," European Journal of Clinical Investigation, vol. 35, no. 4, pp. 236-244, 2005. 
[3] F. Civeira, S. Castillo, R. Alonso et al., "Tendon xanthomas in familial hypercholesterolemia are associated with cardiovascular risk independently of the low-density lipoprotein receptor gene mutation," Arteriosclerosis, Thrombosis, and Vascular Biology, vol. 25, no. 9, pp. 1960-1965, 2005.

[4] D. N. Kiortsis, M. I. Argyropoulou, V. Xydis, S. G. Tsouli, and M. S. Elisaf, "Correlation of achilles tendon thickness evaluated by ultrasonography with carotid intima-media thickness in patients with familial hypercholesterolemia," Atherosclerosis, vol. 186, no. 1, pp. 228-229, 2006.

[5] S. G. Tsouli, D. N. Kiortsis, E. S. Lourida et al., "Autoantibody titers against OxLDL are correlated with Achilles tendon thickness in patients with familial hypercholesterolemia," Journal of Lipid Research, vol. 47, no. 10, pp. 2208-2214, 2006.

[6] M. Hjuler Nielsen, H. Irvine, S. Vedel et al., "Elevated atherosclerosis-related gene expression, monocyte activation and microparticle-release are related to increased lipoprotein-associated oxidative stress in familial hypercholesterolemia," PLoS ONE, vol. 10, no. 4, Article ID e0121516, 2015.

[7] E. Verhoye and M. R. Langlois, "Circulating oxidized lowdensity lipoprotein: a biomarker of atherosclerosis and cardiovascular risk?" Clinical Chemistry and Laboratory Medicine, vol. 47, no. 2, pp. 128-137, 2009.

[8] D. Steinberg and J. L. Witztum, "Is the oxidative modification hypothesis relevant to human atherosclerosis? Do the antioxidant trials conducted to date refute the hypothesis?" Circulation, vol. 105, no. 17, pp. 2107-2111, 2002.

[9] Y. M. Park, "CD36, a scavenger receptor implicated in atherosclerosis," Experimental and Molecular Medicine, vol. 46, no. 6, article e99, 2014.

[10] R. L. Silverstein, "Inflammation, atherosclerosis, and arterial thrombosis: role of the scavenger receptor CD36," Cleveland Clinic Journal of Medicine, vol. 76, no. 2, pp. S27-S30, 2009.

[11] D. Steinberg, "Low density lipoprotein oxidation and its pathobiological significance," Journal of Biological Chemistry, vol. 272, no. 34, pp. 20963-20966, 1997.

[12] K. Chen, M. Febbraio, W. Li, and R. L. Silverstein, "A specific cd36-dependent signaling pathway is required for platelet activation by oxidized low-density lipoprotein," Circulation Research, vol. 102, no. 12, pp. 1512-1519, 2008.

[13] N. Matsumoto, S. Nomura, H. Kamihata, Y. Kimura, and T. Iwasaka, "Increased level of oxidized LDL-dependent monocyte-derived microparticles in acute coronary syndrome," Thrombosis and Haemostasis, vol. 91, no. 1, pp. 146-154, 2004.

[14] H. Wang, Z.-H. Wang, J. Kong et al., "Oxidized low-density lipoprotein-dependent platelet- derived microvesicles trigger procoagulant effects and amplify oxidative stress," Molecular Medicine, vol. 18, no. 2, pp. 159-166, 2012.

[15] V. Combes, A.-C. Simon, G.-E. Grau et al., "In vitro generation of endothelial microparticles and possible prothrombotic activity in patients with lupus anticoagulant," The Journal of Clinical Investigation, vol. 104, no. 1, pp. 93-102, 1999.

[16] M.-L. Liu, M. P. Reilly, P. Casasanto, S. E. McKenzie, and K. J. Williams, "Cholesterol enrichment of human monocyte/ macrophages induces surface exposure of phosphatidylserine and the release of biologically-active tissue factor-positive microvesicles," Arteriosclerosis, Thrombosis, and Vascular Biology, vol. 27, no. 2, pp. 430-435, 2007.

[17] C. Théry, M. Ostrowski, and E. Segura, "Membrane vesicles as conveyors of immune responses," Nature Reviews Immunology, vol. 9, no. 8, pp. 581-593, 2009.
[18] P.-E. Rautou, A.-C. Vion, N. Amabile et al., "Microparticles, vascular function, and atherothrombosis," Circulation Research, vol. 109, no. 5, pp. 593-606, 2011.

[19] A. S. Leroyer, A. Tedgui, and C. M. Boulanger, "Microparticles and type 2 diabetes," Diabetes and Metabolism, vol. 34, supplement 1, pp. S27-S32, 2008.

[20] A. S. Leroyer, A. Tedgui, and C. M. Boulanger, "Role of microparticles in atherothrombosis," Journal of Internal Medicine, vol. 263, no. 5, pp. 528-537, 2008.

[21] C. M. Boulanger, N. Amabile, and A. Tedgui, "Circulating microparticles: a potential prognostic marker for atherosclerotic vascular disease," Hypertension, vol. 48, no. 2, pp. 180-186, 2006.

[22] M. J. VanWijk, E. VanBavel, A. Sturk, and R. Nieuwland, "Microparticles in cardiovascular diseases," Cardiovascular Research, vol. 59, no. 2, pp. 277-287, 2003.

[23] A. Angelillo-Scherrer, "Leukocyte-derived microparticles in vascular homeostasis," Circulation Research, vol. 110, no. 2, pp. 356-369, 2012.

[24] C. M. Boulanger, A. Scoazec, T. Ebrahimian et al., "Circulating microparticles from patients with myocardial infarction cause endothelial dysfunction," Circulation, vol. 104, no. 22, pp. 2649 2652, 2001.

[25] G. N. Chironi, A. Simon, C. M. Boulanger et al., "Circulating microparticles may influence early carotid artery remodeling," Journal of Hypertension, vol. 28, no. 4, pp. 789-796, 2010.

[26] S. Nomura, M. Suzuki, K. Katsura et al., "Platelet-derived microparticles may influence the development of atherosclerosis in diabetes mellitus," Atherosclerosis, vol. 116, no. 2, pp. 235240, 1995.

[27] W. T. Friedewald, R. I. Levy, and D. S. Fredrickson, "Estimation of the concentration of low-density lipoprotein cholesterol in plasma, without use of the preparative ultracentrifuge," Clinical Chemistry, vol. 18, no. 6, pp. 499-502, 1972.

[28] M. H. Nielsen, H. Beck-Nielsen, and M. N. Andersen, "A flow cytometric method for characterization of circulating cellderived microparticles in plasma," Journal of Extracellular Vesicles, vol. 3, Article ID 20795, 2014.

[29] J. Shi, C. W. Heegaard, J. T. Rasmussen, and G. E. Gilbert, "Lactadherin binds selectively to membranes containing phosphatidyl-L-serine and increased curvature," Biochimica et Biophysica Acta-Biomembranes, vol. 1667, no. 1, pp. 82-90, 2004.

[30] J. Shi, Y. Shi, L. N. Waehrens, J. T. Rasmussen, C. W. Heegaard, and G. E. Gilbert, "Lactadherin detects early phosphatidylserine exposure on immortalized leukemia cells undergoing programmed cell death," Cytometry A, vol. 69, no. 12, pp. 1193-1201, 2006.

[31] R. A. Preston, W. Jy, J. J. Jimenez et al., "Effects of severe hypertension on endothelial and platelet microparticles," Hypertension, vol. 41, no. 2, pp. 211-217, 2003.

[32] M. E. Tushuizen, M. Diamant, A. Sturk, and R. Nieuwland, "Cell-derived microparticles in the pathogenesis of cardiovascular disease: friend or foe?" Arteriosclerosis, Thrombosis, and Vascular Biology, vol. 31, no. 1, pp. 4-9, 2011.

[33] C. N. França, M. C. Izar, J. B. Amaral, D. M. Tegani, and F. A. Fonseca, "Microparticles as potential biomarkers of cardiovascular disease," Arquivos Brasileiros de Cardiologia, vol. 104, no. 2, pp. 169-174, 2015.

[34] A. C. Goldberg, P. N. Hopkins, P. P. Toth et al., "Familial hypercholesterolemia: screening, diagnosis and management of pediatric and adult patients: clinical guidance from the National 
Lipid Association Expert Panel on Familial Hypercholesterolemia," Journal of Clinical Lipidology, vol. 5, no. 3, pp. 133-140, 2011.

[35] R. Suades, T. Padró, R. Alonso, P. Mata, and L. Badimon, "Lipidlowering therapy with statins reduces microparticle shedding from endothelium, platelets and inflammatory cells," Thrombosis and Haemostasis, vol. 110, no. 2, pp. 366-377, 2013.

[36] R. Suades, T. Padró, R. Alonso, J. López-Miranda, P. Mata, and L. Badimon, "Circulating CD45+/CD3+ lymphocyte-derived microparticles map lipid-rich atherosclerotic plaques in familial hypercholesterolaemia patients," Thrombosis and Haemostasis, vol. 111, no. 1, pp. 111-121, 2014.

[37] F. Dignat-George and C. M. Boulanger, "The many faces of endothelial microparticles," Arteriosclerosis, Thrombosis, and Vascular Biology, vol. 31, no. 1, pp. 27-33, 2011.

[38] E. J. van Beers, M. C. L. Schaap, R. J. Berckmans et al., "Circulating erythrocyte-derived microparticles are associated with coagulation activation in sickle cell disease," Haematologica, vol. 94, no. 11, pp. 1513-1519, 2009.

[39] R. L. Koshiar, S. Somajo, E. Norström, B. Dahlbäck, and T. Miyata, "Erythrocyte-derived microparticles supporting activated protein c-mediated regulation of blood coagulation," PLoS ONE, vol. 9, no. 8, Article ID e104200, 2014.

[40] A. S. Shet, O. Aras, K. Gupta et al., "Sickle blood contains tissue factor-positive microparticles derived from endothelial cells and monocytes," Blood, vol. 102, no. 7, pp. 2678-2683, 2003.

[41] É. Biró, K. N. Sturk-Maquelin, G. M. T. Vogel et al., "Human cell-derived microparticles promote thrombus formation in vivo in a tissue factor-dependent manner," Journal of Thrombosis and Haemostasis, vol. 1, no. 12, pp. 2561-2568, 2003.

[42] A. P. Owens III and N. MacKman, "Microparticles in hemostasis and thrombosis," Circulation Research, vol. 108, no. 10, pp. 12841297, 2011.

[43] J.-G. Wang, J. C. Williams, B. K. Davis et al., "Monocytic microparticles activate endothelial cells in an IL-1 $\beta$-dependent manner," Blood, vol. 118, no. 8, pp. 2366-2374, 2011.

[44] A. P. Owens III and N. MacKman, "Sources of tissue factor that contribute to thrombosis after rupture of an atherosclerotic plaque," Thrombosis Research, vol. 129, supplement 2, pp. S30S33, 2012.

[45] A. P. Owens III, F. H. Passam, S. Antoniak et al., "Monocyte tissue factor-dependent activation of coagulation in hypercholesterolemic mice and monkeys is inhibited by simvastatin," The Journal of Clinical Investigation, vol. 122, no. 2, pp. 558-568, 2012.

[46] M. B. Fessler, K. Rose, Y. Zhang, R. Jaramillo, and D. C. Zeldin, "Relationship between serum cholesterol and indices of erythrocytes and platelets in the US population," Journal of Lipid Research, vol. 54, no. 11, pp. 3177-3188, 2013.

[47] P. M. Moriarty and C. A. Gibson, "Association between hematological parameters and high-density lipoprotein cholesterol," Current Opinion in Cardiology, vol. 20, no. 4, pp. 318-323, 2005.

[48] A. Blum, "The possible role of red blood cell microvesicles in atherosclerosis," European Journal of Internal Medicine, vol. 20, no. 2, pp. 101-105, 2009.

[49] S. Nomura, A. Shouzu, S. Omoto, M. Nishikawa, T. Iwasaka, and S. Fukuhara, "Activated platelet and oxidized LDL induce endothelial membrane vesiculation: clinical significance of endothelial cell-derived microparticles in patients with type 2 diabetes," Clinical and Applied Thrombosis/Hemostasis, vol. 10, no. 3, pp. 205-215, 2004.
[50] M. Nagahama, S. Nomura, S. Kanazawa, Y. Ozaki, H. Kagawa, and S. Fukuhara, "Significance of anti-oxidized LDL antibody and monocyte-derived microparticles in anti-phospholipid antibody syndrome," Autoimmunity, vol. 36, no. 3, pp. 125-131, 2003.

[51] R. Nergiz-Unal, T. Rademakers, J. M. M. Cosemans, and J. W. M. Heemskerk, " $\mathrm{Cd} 36$ as a multiple-ligand signaling receptor in atherothrombosis," Cardiovascular and Hematological Agents in Medicinal Chemistry, vol. 9, no. 1, pp. 42-55, 2011.

[52] R. Nergiz-Unal, M. M. E. Lamers, R. Van Kruchten et al., "Signaling role of CD36 in platelet activation and thrombus formation on immobilized thrombospondin or oxidized lowdensity lipoprotein," Journal of Thrombosis and Haemostasis, vol. 9, no. 9, pp. 1835-1846, 2011.

[53] R. Lacroix, S. Robert, P. Poncelet, R. S. Kasthuri, N. S. Key, and F. Dignat-George, "Standardization of platelet-derived microparticle enumeration by flow cytometry with calibrated beads: results of the International Society on Thrombosis and Haemostasis SSC Collaborative workshop," Journal of Thrombosis and Haemostasis, vol. 8, no. 11, pp. 2571-2574, 2010.

[54] R. Lacroix, S. Robert, P. Poncelet, and F. Dignat-George, "Overcoming limitations of microparticle measurement by flow cytometry," Seminars in Thrombosis and Hemostasis, vol. 36, no. 8, pp. 807-818, 2010.

[55] E. van der Pol, M. J. C. van Gemert, A. Sturk, R. Nieuwland, and T. G. van Leeuwen, "Single vs. swarm detection of microparticles and exosomes by flow cytometry," Journal of Thrombosis and Haemostasis, vol. 10, no. 5, pp. 919-930, 2012. 


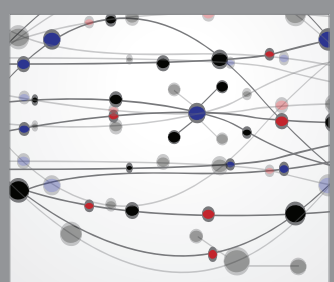

The Scientific World Journal
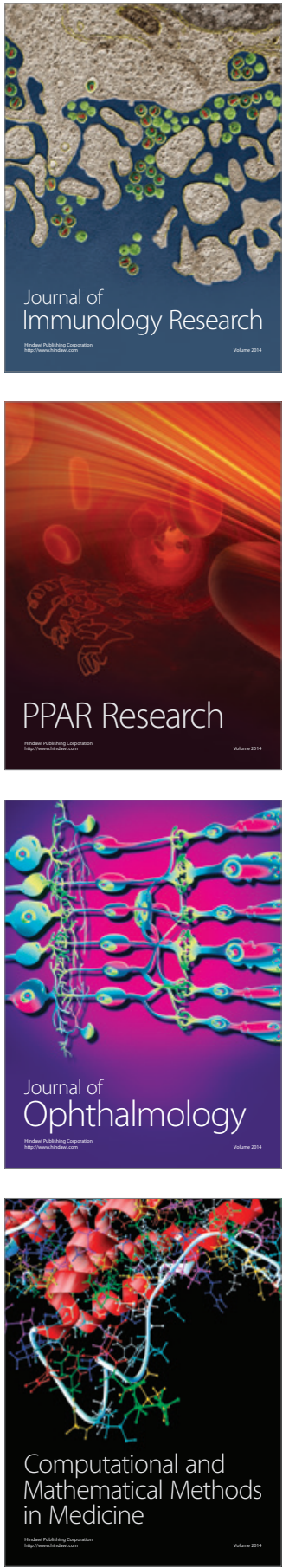

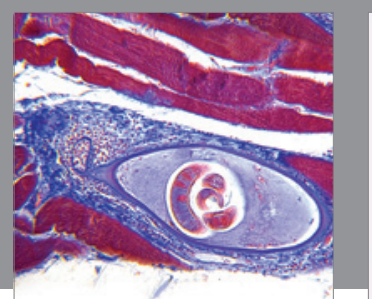

Gastroenterology Research and Practice

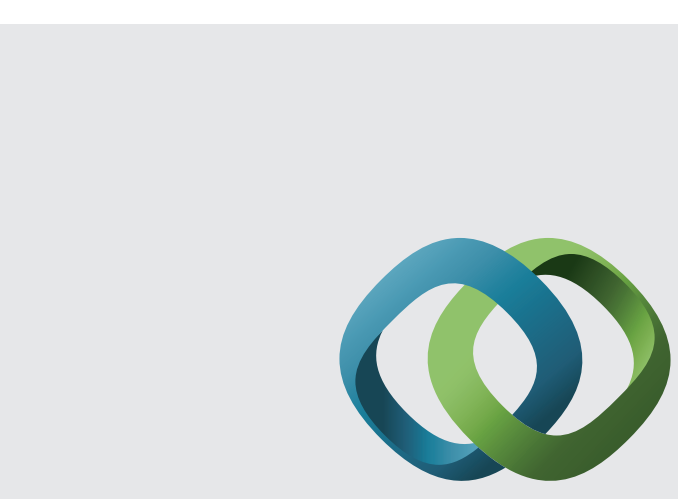

\section{Hindawi}

Submit your manuscripts at

http://www.hindawi.com
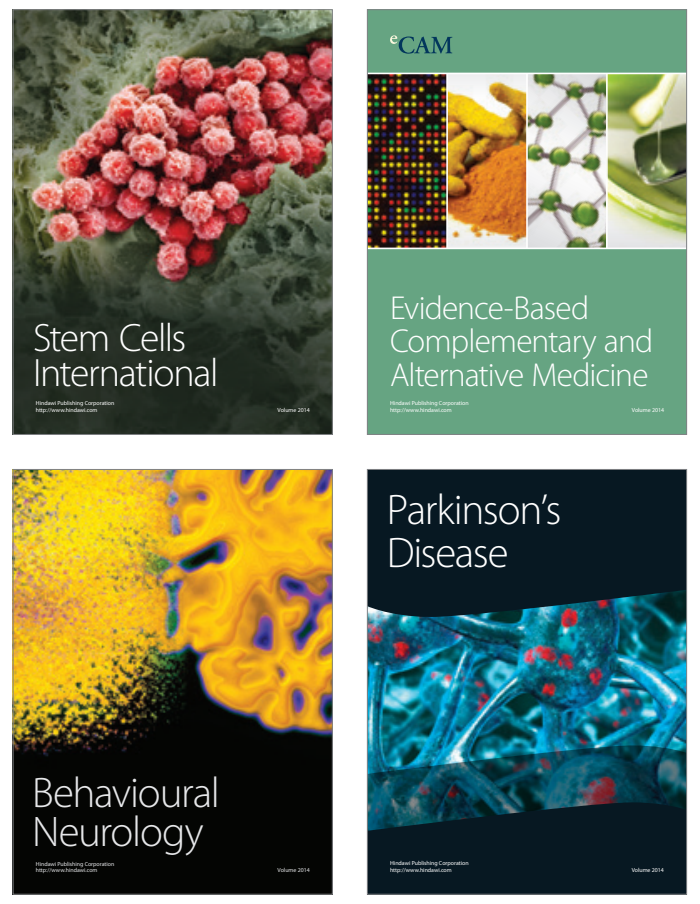
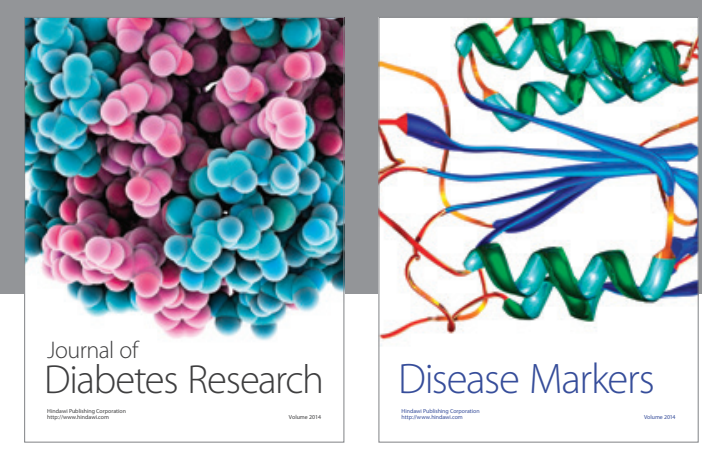

Disease Markers
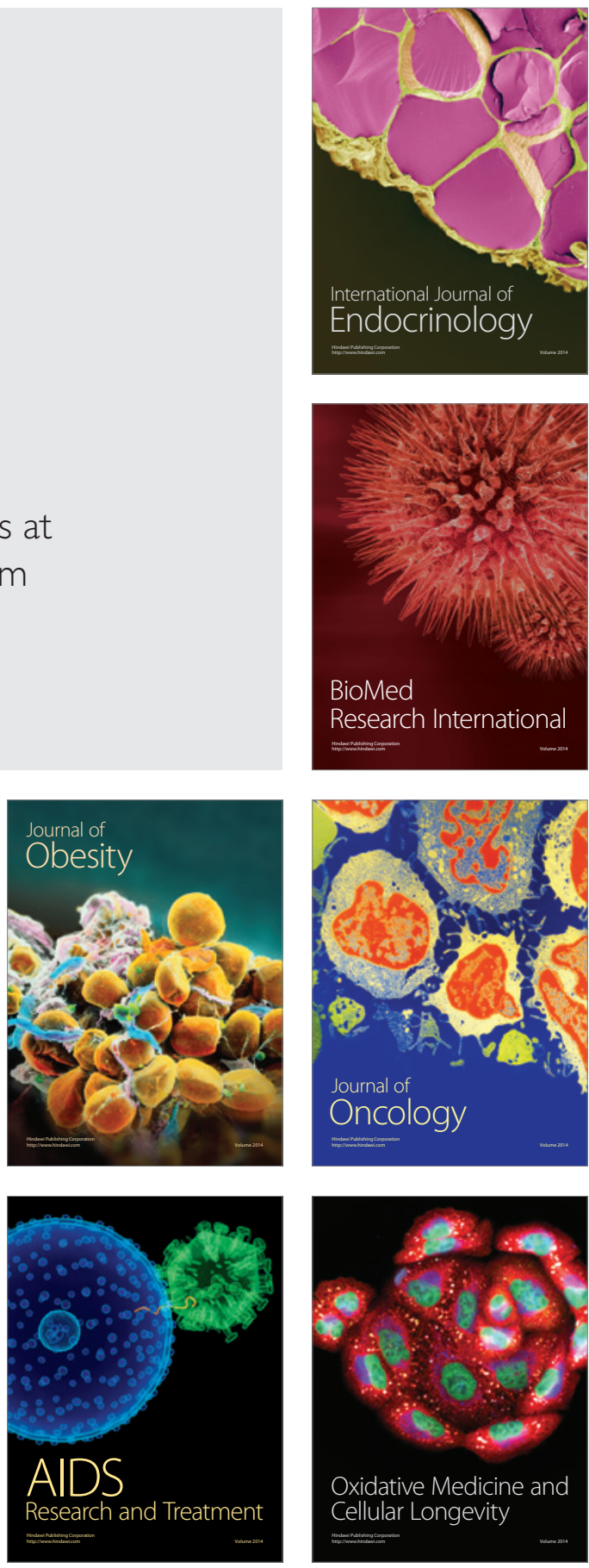